"average or larger" to represent low birth weight and normal birth weight respectively. The fuel type was classified as high pollution, and low pollution. Univariate and multivariate logistic regression models were developed using SURVEYLOGISTIC procedure in SAS system in which child factors, maternal factors and demographic factors were added step-by-step to the main exposure variable.

Results Babies not weighed at birth in households using high and low pollution fuels was $71.4 \%$ and $26.1 \%$ respectively. Missing information on size at birth was only $6.4 \%$. The results for association between high pollution fuels use and low birth weight were as follows: univariate OR was 1.41 (95\% CI 1.27 to 1.55). Adjusted ORs in models $1,2,3$ were $1.41(1.29,1.57), 1.21$ (1.06, 1.32), 1.07 $(0.94,1.22)$ respectively. Gender, birth order, mother's BMI, haemoglobin level and education were significant throughout. In model 3 , there was no association between birth weight and fuel type but wealth index and religion were significant.

Conclusion Our results are consistent with previous observational studies. The effect size of association between size at birth and exposure to biomass smoke during pregnancy was small and was masked by wealth index and religion.

\section{P2-477 THE ASSOCIATION OF ADIPONECTIN GENE POLYMORPHISMS (+45T>G AND + 276G $>$ T) WITH ADIPONECTIN LEVELS BUT NOT WITH CHARACTERISTICS OF THE INSULIN RESISTANCE SYNDROME IN NORTHERN INDIA}

doi:10.1136/jech.2011.142976m.5

${ }^{1} \mathrm{~N}$ Srivastava, ${ }^{1} \mathrm{~J}$ Prakash, ${ }^{1} \mathrm{C}$ G Agarwal, 'B Mittal. ${ }^{1} \mathrm{KGMU}$, Lucknow, Uttar Pradesh, India; ${ }^{2}$ SGPGIMS, Lucknow, Uttar Pradesh, India

Introduction Adiponectin, the major adipocyte secretary protein, improves insulin sensitivity and possesses anti-inflammatory and anti atherosclerotic properties. In the present study, we investigated the association of adiponectin gene (APM1) +45T>G (rs2241766) and $+276 \mathrm{G}>\mathrm{T}$ (rs1501299) polymorphisms with serum adiponectin levels and metabolic parameters of the insulin resistance syndrome, in northern Indian population.

Methods A case control study was conducted with 642 subjects (309 obese subjects $(B M I \geq 30)$ and 333 non-obese $(B M I<30)$ subjects) to examine the association of adiponectin gene (APM1) polymorphisms with serum adiponectin levels and other metabolic parameters in northern Indian population. All subjects were genotyped through PCR-RFLP. Insulin, leptin, lipid profile and glucose levels were estimated using standard protocols.

Results The variant G-allele of SNP $+45 \mathrm{~T}>\mathrm{G}$ and T-allele of SNP +276G $>$ T were associated with significantly higher serum adiponectin levels ( $p=0.0001$ and $p=0.0001$, respectively). The most frequent haplotype TG defined by both loci showed a strong association with lower serum adiponectin concentrations, higher levels of insulin, and HOMA-IR index.

Conclusion We observed a strong association of the APM1 45-276 genotypes and TG (rs2241766, rs1501299) haplotypes with adiponectin levels in healthy northern Indians. The TG haplotype associated with higher levels of insulin, HOMA-IR index, and lower serum adiponectin concentrations.

\section{P2-478 WITHDRAWN}

\section{P2-479 DETERMINANTS OF FUNCTIONING AND DISABILITY IN AGED PERSONS: RESULTS FROM THE KORA-AGE STUDY}

doi:10.1136/jech.2011.142976m.6

${ }^{1} \mathrm{R}$ Strobl, ${ }^{*} \mathrm{M}$ Müller, ${ }^{2} \mathrm{~A}$ Döring, ${ }^{1} \mathrm{E}$ Grill. ${ }^{1}$ Institute for Health and Rehabilitation Sciences, Ludwig-Maximilians-Universität München, Munich, Germany; ${ }^{2}$ Institute of
Epidemiology, Helmholtz Zentrum München, German Research Center for Environmental Health, Neuherberg, Germany

Introduction Increasing attention is paid on functioning and disability as determinants of autonomy and independent living in aged persons. Yet, determinants of functioning and disability have not been studied extensively in this age group. Most studies took a disease-oriented approach by investigating populations with specific health conditions. So far, few representative findings on the prevalence of disability and its determinants in aged persons are available. The objective of our study is to examine distribution and determinants of functioning and disability in aged persons in the community.

Methods The data originate from the MONICA/KORA study, a population-based cohort study established in 1984 in Germany. Survivors of the original cohorts aged 65 years and above were examined from $01 / 2009$ to $12 / 2009$. Disability was assessed with the Health Assessment Questionnaire. Logistic regression was used to estimate raw effects and conditional inference trees with Bonferroni adjusted p-values were used to identify high risk groups. Results We analysed a total of 4127 individuals (51.2\% female) with a mean age of 73.3 years ( $\mathrm{SD}=6.1)$. Disability was present in $44.7 \%$ of all individuals (54\% for women, $35 \%$ for men). Prevalence of disability increased from $28.8 \%$ for $65-70$ year old individuals to $75.9 \%$ for individuals over 80 . Prevalence was $31.6 \%$ in individuals without comorbidity, else $55.9 \%(R R=1.77)$. High risk groups were found among all age groups.

Conclusion Minimal disability is highly prevalent in aged persons. The results give a representative overview of the prevalence and determinants of disability in the aged in the German population.

\section{P2-480 INTERNATIONAL COMPARISON ON THE VALIDITY AND THE RELIABILITY OF THE "INTERNATIONAL AIDS QUESTIONNAIRE"}

doi:10.1136/jech.2011.142976m.7

${ }^{1} \mathrm{M}$ Takahashi, ${ }^{1} \mathrm{M}$ Doi, ${ }^{1} \mathrm{M}$ Okada, ${ }^{2} \mathrm{~T}$ Munakata, ${ }^{1} \mathrm{Y}$ Wagatsuma. ${ }^{1}$ Department of Epidemiology, Graduate School of Comprehensive Human Sciences, University of Tsukuba, Tsukuba, Japan; ${ }^{2}$ Department of Human Care Sciences, Graduate School of Comprehensive Human Sciences, University of Tsukuba, Tsukuba, Japan

Introduction "International AIDS Questionnaire (IAQ)" was originally proposed by Davis in 1998 and has been used in Australia, China and the U.S. The IAO enables to assess adolescent's awareness to HIV/ AIDS by four factors; (1) misconceptions about HIV transmission, (2) prejudice/attitudes towards infected people, (3) personal risks and (4) factual knowledge about HIV/AIDS. The objective of this study was to validate the IAO among Japanese adolescents.

Methods We developed the "International AIDS Questionnaire Japanese Version (IAQ-J)" by using back translation technique. IAQ-J was assessed on a sample of national high school students and private high school students from Eastern Japan. We conducted Exploratory and Confirmatory Factor Analysis and calculated Cronbach's $\alpha$ for total and each factor.

Results The results indicated that four-factor model was suitable to IAQ-J (GFI=0.903, AGFI=0.872). This result coincides to Chinese and English versions of IAQ. Total Cronbach's $\alpha$ of IAQ-J was 0.79 . However, Cronbach's $\alpha$ for the "attitudes" factor was 0.66 , indicating less reliable results. More than $70 \%$ of the students answered "no idea" to one question from the "attitudes" factor. After the exclusion of this item, Cronbach's $\alpha$ of the "attitudes" factor was raised to 0.75 . This may indicate that this question was not appropriate for Japanese. Cronbach's $\alpha$ of the "factual knowledge" of IAQ-J was higher than other language versions of IAQ.

Conclusion IAQ is a simple and useful tool to assess the awareness on HIV/AIDS among adolescents. We recommend more language versions are tested and shared for international studies to prevent HIV/AIDS. 